\title{
Funneling of Electromagnetic Energy Through a Rectangular Hole
}

\author{
Ji-Hun KANG and Q-Han PARK* \\ Department of Physics, Korea University, Seoul 136-701 \\ Dai Sik KIM \\ School of Physics, Seoul National University, Seoul 151-747
}

(Received 25 August 2008)

\begin{abstract}
When light passes through a subwavelength-scale square hole in a perfect conductor, the flow of elecrtromagnetic energy exhibits a strikingly rich behavior that depends on the geometry of the rectangular hole. We present a detailed calculation and demonstrate that funneling of electromagnetic energy occurs when certain resonance conditions are met.
\end{abstract}

PACS numbers: 42.25.-p, 42.82.Et, 42.87.-d

Keywords: Funneling, Rectangular hole, Resonance, Plasmonics

\section{INTRODUCTION}

Strong excitation of a electric field is generally accompanied by a strong build up of electric charge as in the case of a charged capacitor [1]. In the presence of incident electromagnetic waves, electric currents are induced on a metal surface, and charges can be accumulated at the junction blocking the flow of current. Strong build up of charge is expected when the junction has a sharp edge or more importantly when the system is in resonance with the exciting external source. Recently, localized plasmons in nano-scale metallic structures have witnessed an explosion of research efforts with applications in various fields of science, including physics [2-5], chemistry, and biomedical sciences [6-9]. Enhanced transmission of light through holes that are periodically perforated in a metal film demonstrated by Ebbesen, heralded many of these plasmonic efforts [10]. The physical origin of enhanced transmission is the excitation of surface plasmons coupled to incoming and outgoing radiations that are also in resonance with the periodic structures $[2,4$, 11-14]. In the case of the Ebbesen structure, the holes are too small to support propagating mode, and light passes through the holes only via a tunneling process. When the size of the hole becomes larger, of the order of the wavelength, propagating modes become available, and a single hole alone can admit resonance. The singlehole resonance and subsequent enhanced transmission is equally important in surface plasmon applications.

In this paper, we consider a single rectangular hole and provide a detailed analysis of the field enhancement. We show that resonance occurs when the long side of a

*E-mail: qpark@korea.ac.kr; Fax: +82-2-927-3292 rectangle is about half the wavelength. The dependence of the enhancement on the size of the rectangle and on the thickness of metal film is obtained, and the nature of the resonance is clarified.

\section{FIELD ENHANCEMENT}

Consider a metal film with a rectangular hole of size $a \times b$, as shown in Fig. 1, and TM-polarized light incident on it. Field configurations can be obtained by solving Maxwell's equation with appropriate boundary conditions imposed. For simplicity, we assume that metal to be a perfect conductor and impose a vanishing boundary condition for the tangential component of the electric field. Note that TM-polarized light incident obliquely on a metal film with rectangular holes couples to the TE modes of the rectangular holes. Inside the hole $(-h / 2<z<h / 2$, which we call region II), we make a single-mode approximation and take into account only the lowest $T E_{01}$ mode inside a rectangular hole of size

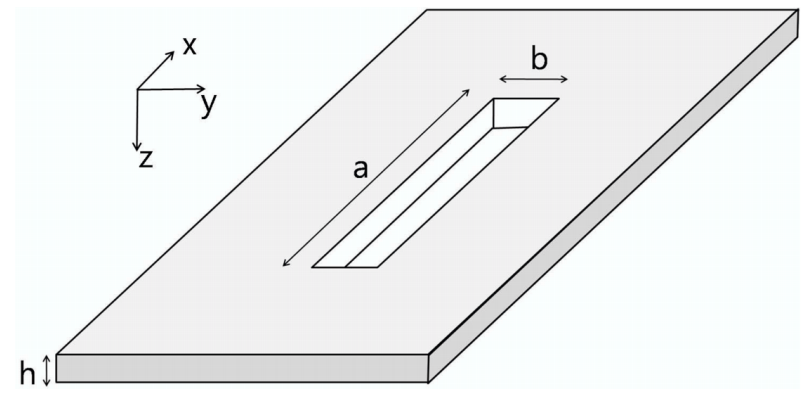

Fig. 1. A metal film with a rectangular hole of size $a \times b$. 


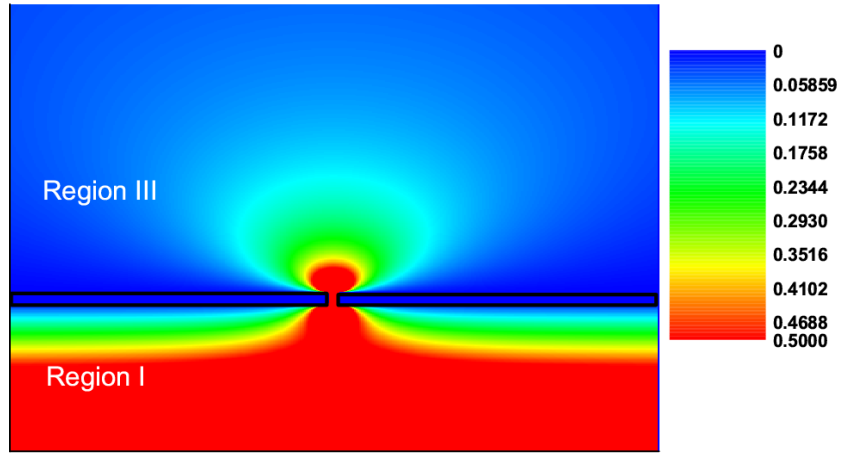

Fig. 2. (Color online) Cross-sectional distribution of the electric field near a rectangular hole of $a=\lambda / 2, b=\lambda / 60$, and $h=\lambda / 30$, calculated by using a finite-difference timedomain (FDTD) method, for light incident with the resonant wavelength $\lambda$.

$$
\begin{aligned}
a \times b(a & >b), \text { as given by } \\
E_{x}^{I I} & =0, \\
E_{y}^{I I} & =\sin \left(\frac{\pi x}{a}\right)\left[-E_{L} \frac{\cos (\mu z)}{\sin (\mu h / 2)}+E_{R} \frac{\sin (\mu z)}{\cos (\mu h / 2)}\right] \\
E_{z}^{I I} & =0 \\
H_{x}^{I I} & =\frac{i \mu}{k_{0}} \sqrt{\frac{\epsilon_{0}}{\mu_{0}}} \sin \left(\frac{\pi x}{a}\right) \\
\times & {\left[E_{L} \frac{\sin (\mu z)}{\sin (\mu h / 2)}+E_{R} \frac{\cos (\mu z)}{\cos (\mu h / 2)}\right] } \\
H_{y}^{I I} & =0 \\
H_{z}^{I I} & =-\frac{i \pi}{k_{0} a} \sqrt{\frac{\epsilon_{0}}{\mu_{0}}} \cos \left(\frac{\pi x}{a}\right) \\
\times & {\left[-E_{L} \frac{\cos (\mu z)}{\sin (\mu h / 2)}+E_{R} \frac{\sin (\mu z)}{\cos (\mu h / 2)}\right] }
\end{aligned}
$$

where $\mu=\sqrt{k_{0}^{2}-(\pi / a)^{2}}$.

The incoming TM-polarized light and the reflected light in the region $z>h / 2$ (which we call region $\mathrm{I}$ in Fig. 2) can be expressed as follows:

$$
\begin{aligned}
H_{x}^{I} & =\sqrt{\frac{\epsilon_{0}}{\mu_{0}}} \int d k_{x} d k_{y}\left[g_{x}\left(k_{x}, k_{y}\right) e^{i \Theta+i k_{z}(z-h / 2)}\right. \\
& \left.+\delta\left(k_{x}\right) \delta\left(k_{y}\right) e^{-i k_{z}(z-h / 2)}\right], \\
H_{y}^{I} & =\sqrt{\frac{\epsilon_{0}}{\mu_{0}}} \int d k_{x} d k_{y}\left[g_{y}\left(k_{x}, k_{y}\right) e^{i \Theta+i k_{z}(z-h / 2)}\right], \\
H_{z}^{I} & =\sqrt{\frac{\epsilon_{0}}{\mu_{0}}} \int d k_{x} d k_{y}\left[g_{z}\left(k_{x}, k_{y}\right) e^{i \Theta+i k_{z}(z-h / 2)}\right],
\end{aligned}
$$

where

$$
\begin{aligned}
& \Theta=k_{x}(x-a / 2)+k_{y}(y-b / 2), \\
& k_{z}^{2}=k_{0}^{2}-k_{x}^{2}-k_{y}^{2} .
\end{aligned}
$$

The sign of $k_{z}$ is chosen such that the imaginary part of $k_{z}$ is positive. In the same manner, we take the expressions for the transmitted light in the region $z<-h / 2$ (region III) to be

$$
\begin{aligned}
& H_{x}^{I I I}=\sqrt{\frac{\epsilon_{0}}{\mu_{0}}} \int d k_{x} d k_{y}\left[f_{x}\left(k_{x}, k_{y}\right) e^{\left.i \Theta-i k_{z}(z+h / 2)\right],}\right. \\
& H_{y}^{I I I}=\sqrt{\frac{\epsilon_{0}}{\mu_{0}}} \int d k_{x} d k_{y}\left[f_{y}\left(k_{x}, k_{y}\right) e^{\left.i \Theta-i k_{z}(z+h / 2)\right],}\right. \\
& H_{z}^{I I I}=\sqrt{\frac{\epsilon_{0}}{\mu_{0}}} \int d k_{x} d k_{y}\left[f_{z}\left(k_{x}, k_{y}\right) e^{i \Theta-i k_{z}(z+h / 2)}\right] .
\end{aligned}
$$

With field configurations in each region satisfying Maxwell's equation separately, the overall solution is obtained by matching the boundary conditions at the metal surface and at the interface $z= \pm h / 2$. The vanishing tangential electric field on a metal surface requires

$$
\begin{aligned}
& \nabla \times\left.\vec{H}\right|_{\text {metal }}=\left.0 \rightarrow\left(\frac{\partial H_{z}}{\partial y}-\frac{\partial H_{y}}{\partial z}\right)\right|_{\text {metal }}=0, \\
& \left.\left(\frac{\partial H_{x}}{\partial z}-\frac{\partial H_{z}}{\partial x}\right)\right|_{\text {metal }}=0
\end{aligned}
$$

so that

$$
\begin{aligned}
& \int d k_{x} d k_{y}\left(i k_{y} f_{z}+i k_{z} f_{y}\right) e^{i \Theta}=0, \\
& \int d k_{x} d k_{y}\left(i k_{y} g_{z}-i k_{z} g_{y}\right) e^{i \Theta}=0 .
\end{aligned}
$$

The $y$-component of the electric field vanishes on a metal surface and matches with the internal mode at the interface such that

$$
\begin{aligned}
& \int d k_{x} d k_{y}\left(i k_{z} f_{x}+i k_{x} f_{z}\right) e^{i \Theta} \\
& =-i k_{0} \sin \left(\frac{\pi x}{a}\right)\left[E_{L} \cot (\mu h / 2)+E_{R} \tan (\mu h / 2)\right] \\
& =0 \text { otherwise } \\
& \int d k_{x} d k_{y}\left[i k_{z} g_{x}-i k_{0} \delta\left(k_{x}\right) \delta\left(k_{y}\right)-i k_{x} g_{z}\right] e^{i \Theta} \\
& =i k_{0} \sin \left(\frac{\pi x}{a}\right)\left[E_{L} \cot (\mu h / 2)-E_{R} \tan (\mu h / 2)\right] \\
& =0 \text { otherwise } .
\end{aligned}
$$

The divergencelessness of $\vec{H}(\nabla \cdot \vec{H}=0)$ gives rise to

$$
\begin{aligned}
& f_{z}=\frac{1}{k_{z}}\left(k_{x} f_{x}+k_{y} f_{y}\right)=\frac{k_{x} k_{z}}{k_{y}^{2}+k_{z}^{2}} f_{x}, \\
& f_{y}=-\frac{k_{x} k_{y}}{k_{y}^{2}+k_{z}^{2}} f_{x}, \\
& g_{z}=-\frac{1}{k_{z}}\left(k_{x} g_{x}+k_{y} g_{y}\right)=-\frac{k_{x} k_{z} g_{x}}{k_{z}^{2}+k_{y}^{2}}, \\
& g_{y}=-\frac{k_{x} k_{y} g_{x}}{k_{z}^{2}+k_{y}^{2}} .
\end{aligned}
$$

On the other hand, the continuity of $\vec{H}$ at the interface requires that

$$
\begin{aligned}
& \frac{i \mu}{k_{0}} \sin \left(\frac{\pi x}{a}\right)\left[-E_{L}+E_{R}\right]=\int d k_{x} d k_{y} f_{x} e^{i \Theta} \\
& \frac{i \mu}{k_{0}} \sin \left(\frac{\pi x}{a}\right)\left[E_{L}+E_{R}\right]=\int d k_{x} d k_{y} g_{x} e^{i \Theta}+1
\end{aligned}
$$


which, integrating over $x$ after multiplying by the factor $\sin (\pi x / a)$, can be converted to

$$
\begin{aligned}
& \frac{i \mu}{k_{0}} \frac{a b}{2}\left[-E_{L}+E_{R}\right]=\int d k_{x} d k_{y} f_{x} \frac{a b}{2} J, \\
& \frac{i \mu}{k_{0}} \frac{a b}{2}\left[E_{L}+E_{R}\right]=\int d k_{x} d k_{y} g_{x} \frac{a b}{2} J+\frac{2 a b}{\pi}, \\
& J=\frac{2}{a b} \int_{0}^{a} d x \int_{0}^{b} d y \sin \left(\frac{\pi x}{a}\right) e^{i \Theta} .
\end{aligned}
$$

Similarly, by taking the inverse Fourier transform of Eq. $(7)$, we convert $f_{x}$ and $g_{x}$ :

$$
\begin{aligned}
f_{x} & =-\frac{k_{y}^{2}+k_{z}^{2}}{k_{0} k_{z}} K\left[E_{L} \cot (\mu h / 2)+E_{R} \tan (\mu h / 2)\right], \\
g_{x} & =\frac{k_{y}^{2}+k_{z}^{2}}{k_{0} k_{z}}\left[\delta\left(k_{x}\right) \delta\left(k_{y}\right)\right. \\
& \left.+K\left(E_{L} \cot (\mu h / 2)-E_{R} \tan (\mu h / 2)\right)\right], \\
K & =\frac{1}{4 \pi^{2}} \int_{0}^{a} d x \int_{0}^{b} d y \sin \left(\frac{\pi x}{a}\right) e^{-i \Theta} .
\end{aligned}
$$

Combing them with Eq. (10), we finally solve for $E_{L}$ and $E_{R}$ :

$$
\begin{aligned}
& E_{L}=\frac{1}{D}\left[\mu \sin (\mu h)-2 i k_{0} W \sin ^{2}(\mu h / 2)\right], \\
& E_{R}=\frac{1}{D}\left[\mu \sin (\mu h)+2 i k_{0} W \cos ^{2}(\mu h / 2)\right],
\end{aligned}
$$

where

$$
\begin{aligned}
D & =\frac{i \pi}{4 k_{0}}\left[\sin (\mu h)\left(\mu^{2}+k_{0}^{2} W^{2}\right)+2 i \mu k_{0} W \cos (\mu h)\right], \\
W & =\int d k_{x} d k_{y} \frac{k_{y}^{2}+k_{z}^{2}}{k_{0} k_{z}} \frac{a b}{8 \pi^{2}} J^{2} \approx \frac{32 a b}{3 \pi} k_{0}^{2}, \\
J & =\frac{2}{a b} \int_{0}^{a} d x \int_{0}^{b} d y \sin \left(\frac{\pi x}{a}\right) e^{i \Theta} \\
& =\operatorname{sinc}\left(b k_{y} / 2\right)\left[\operatorname{sinc}\left(\pi / 2+a k_{x} / 2\right)\right. \\
& \left.+\operatorname{sinc}\left(\pi / 2-a k_{x} / 2\right)\right] .
\end{aligned}
$$

\section{FUNNELING OF THE ELECTROMAGNETIC FIELD}

Having determined all relevant coefficients, the solution of Maxwell's equation for TM light incident on a rectangular hole is uniquely found up to an overall constant characterizing the strength of the incoming wave. In particular, the electromagnetic field inside the rectangular hole is given by

$$
\begin{aligned}
E_{y} & =-\frac{8 k_{0}}{i \mu \pi} \sin \left(\frac{\pi x}{a}\right) \\
& \times \frac{i \mu \cos (\mu z+\mu h / 2)+k_{0} W \sin (\mu z+\mu h / 2)}{i \mu\left(1+\frac{k_{0}^{2} W^{2}}{\mu^{2}}\right) \sin (\mu h)-2 k_{0} W \cos (\mu h)},
\end{aligned}
$$

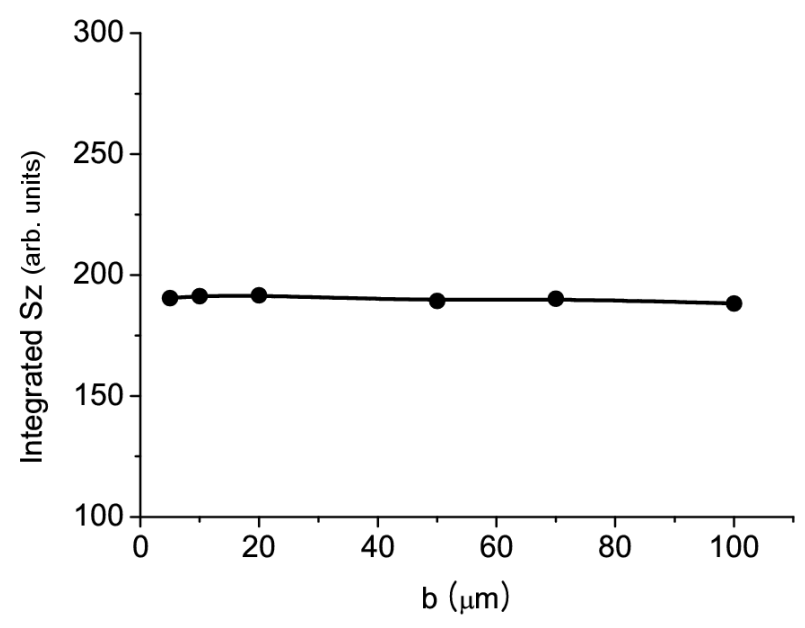

Fig. 3. Result from a FDTD calculation showing the Poynting vector, $\mathrm{Sz}$, integrated over the area of the hole at the resonant wavelength. For a simple case, $a$ and $h$ are set to $100 \mu \mathrm{m}$ and $1 \mu \mathrm{m}$, respectively.

$$
\begin{aligned}
H_{x} & =\frac{8}{\pi} \sqrt{\frac{\epsilon_{0}}{\mu_{0}}} \sin \left(\frac{\pi x}{a}\right) \\
\times & \frac{i \mu \sin (\mu z+\mu h / 2)-k_{0} W \cos (\mu z+\mu h / 2)}{i \mu\left(1+\frac{k_{0}^{2} W^{2}}{\mu^{2}}\right) \sin (\mu h)-2 k_{0} W \cos (\mu h)} .
\end{aligned}
$$

These fields determine the electromagnetic energy flow in terms of the Poynting vector

$$
\begin{aligned}
S_{z} & =\frac{1}{2} \operatorname{Re}\left(E_{x} H_{y}^{*}-E_{y} H_{x}^{*}\right)=-\frac{1}{2} \operatorname{Re}\left(E_{y} H_{x}^{*}\right) \\
& =\frac{1}{2} \operatorname{Re}\left[\frac{i \mu}{k_{0}} \sqrt{\frac{\epsilon_{0}}{\mu_{0}}} \sin ^{2}\left(\frac{\pi x}{a}\right) \frac{E_{L}^{*} E_{R}-E_{L} E_{R}^{*}}{\sin (\mu h)}\right] \quad(14) \\
& =-\frac{16}{\pi^{2}} \frac{\mu^{2} \sqrt{\frac{\epsilon_{0}}{\mu_{0}}} \sin ^{2}\left(\frac{\pi x}{a}\right) k_{0}^{2}\left(W+W^{*}\right)}{\sin (\mu h)\left(\mu^{2}+k_{0}^{2} W^{2}\right)+\left.2 i \mu k_{0} W \cos (\mu h)\right|^{2}} .
\end{aligned}
$$

This is the main result of the present paper. Note that the Poynting vector $S_{z}$ is independent of the longitudinal direction $z$, which is consistent with constant energy flow along the longitudinal direction. When $\mu$ is small $(\mu \ll$ $\left.k_{0}\right)$, the total energy flow is

$$
\begin{aligned}
E_{t o t} & =\int_{0}^{a} d x \int_{0}^{b} d y S_{z} \\
& \approx-\frac{8}{\pi^{2}} \sqrt{\frac{\epsilon_{0}}{\mu_{0}}} \frac{\mu^{2} a b k_{0}^{2}\left(W+W^{*}\right)}{\mu h\left(\mu^{2}+k_{0}^{2} W^{2}\right)+\left.2 i \mu k_{0} W\right|^{2}} \\
& \approx-\frac{512}{3 \pi^{3}} \sqrt{\frac{\epsilon_{0}}{\mu_{0}}} \frac{a^{2} b^{2} k_{0}^{4}}{h^{2}\left[k_{0}^{2}-\pi^{2} / a^{2}\right]^{2}+\left[64 a b k_{0}^{3} / 3 \pi\right]^{2}} .
\end{aligned}
$$

This shows that the transmitted energy reaches maximum near the half wavelength resonance $k_{0} \approx \pi / a$. Near the resonance,

$$
E_{t o t} \approx-\frac{512}{3 \pi^{3}} \sqrt{\frac{\epsilon_{0}}{\mu_{0}}} \frac{a^{2} b^{2} k_{0}^{4}}{\left[64 a b k_{0}^{3} / 3 \pi\right]^{2}}=-\frac{3}{8 \pi} \sqrt{\frac{\epsilon_{0}}{\mu_{0}}} \frac{1}{k_{0}^{2}} .
$$

This shows a striking result that the total energy remains constant irrespective of the size of the area $a b$. That is, 


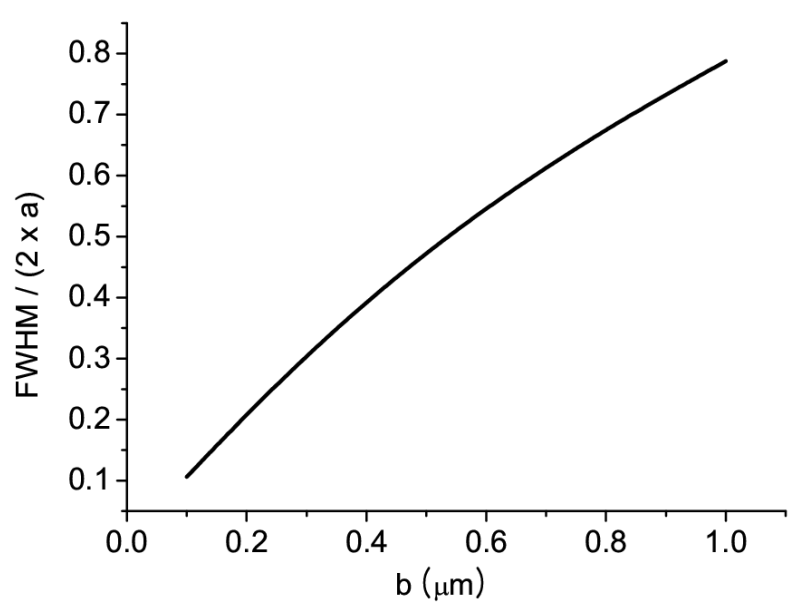

Fig. 4. FWHM of the spectrum of total energy flow from a hole with $a=100 \mu \mathrm{m}$ and $h=20 \mu \mathrm{m}$.

the total energy does not change, not even with decreasing width $b$. This fact is consistent with the results from the FDTD calculation, as shown in Fig. 3. Thus, electromagnetic energy, indeed, funnels through a rectangular hole near resonance.

Finally, we point out that the spectrum of the total energy flow is narrower with small $a$, as Eq. (16) and (17) predict. Shown in Fig. 4 is the full width at half maximum (FWHM) of the spectrum of the total energy flow from a hole, which shows that a small $b$ yields a narrower spectrum.

\section{CONCLUSION}

In conclusion, we have shown that resonant funneling of an electromagnetic wave occurs when the wavelength is about twice as long as the long side of a rectangular hole. The energy transmitted through the rectangular hole at the resonant wavelength remains constant and is independent of the size of the hole.

\section{ACKNOWLEDGMENTS}

This work is supported by the q-Psi, the Korea Science and Engineering Foundation (KOSEF), the Korea Research Foundation (KRF), a National Science \& Technology Scholarship, and the Seoul R\&BD Program.

\section{REFERENCES}

[1] Y. M. Strelniker and D. J. Bergman, Phys. Rev. B 59, 12763 (1999).

[2] J. B. Pendry, L. Martin-Moreno and F. J. Garcia-Vidal, Science 305, 847 (2004)

[3] A. G. Brolo, E. Arctander, R. Gordon, B. Leathem and K. L. Kavanagh, Nano Lett. 4, 2015 (2004).

[4] Q.-H. Park, K. G. Lee and D. S. Kim, J. Korean Phys. Soc. 49, 2075 (2006).

[5] S. B. Choi, D. J. Park, D. S. Kim, M. S. Jeong and C. C. Byeon, J. Korean Phys. Soc. 53, 713 (2008).

[6] L. Novotny, D. W. Pohl and B. Hecht, Opt. Lett. 20, 970 (1995).

[7] B. Hecht, B. Sick, U. P. Wild, V. Deckert, R. Zenobi, O. J. F. Martin and D. W. Pohl, J. Chem. Phys. 112, 7761 (2000).

[8] J. T. Bahns, F. Yan, D. Qiu, R. Wang and L. Chen, Appl. Spectrosc. 60, 989 (2006).

[9] R. S. Decca, H. D. Drew and K. L. Empson, Appl. Phys. Lett. 70, 61 (1997).

[10] T. W. Ebbesen, H. J. Lezec, H. F. Ghaemi, T. Thio and P. A. Wolff, Nature (London) 391, 667 (1998).

[11] D. J. Park, S. B. Choi, K. J. Ahn and D. S. Kim, Phys. Rev. B 77, 115451 (2008).

[12] J. W. Lee, M. A. Seo, D. S. Kim, S. C. Jeoung, C. Lienau, J. H. Kang and Q. H. Park, Appl. Phys. Lett. 88, 071114 (2006).

[13] L. Martin-Moreno, F. J. Garcia-Vidal, H. J. Lezec, K. M. Pellerin, T. Thio, J. B. Pendry and T. W. Ebbesen, Phys. Rev. Lett. 86, 1114 (2001).

[14] Z. Liu, S. Durant, H. Lee, Y. Pikus, N. Fang, Y. Xiong, C. Sun and X. Zhang, Nano Lett. 7, 403 (2007). 